\title{
Editorial
}

\section{Special Issue On New Trends In Signal Processing And Electronics Engineering}

\author{
Jin-bao Li ${ }^{1, \S}$, Shou-xu Jiang ${ }^{2, \#}$ and Chunxiang Zhang ${ }^{2, \#}$ \\ ${ }^{1}$ Computer School of Harbin Institute of Technology, China \\ ${ }^{2}$ Software School of Harbin University of Science and Technology, China
}

Welcome to this special issue on New Trends in Signal Processing and Electronics Engineering which comprises 7 papers selected from the 2012 International Conference on Information, Computing and Telecommunications (ICICT 2012) held on Jan 7 8, 2012 in Harbin, P.R. China. This conference was sponsored and organized by Harbin University of Science and Technology, with the collaboration of International Science and Engineering Research Center. The conference was technically co-sponsored by Harbin Engineering University, Northeast Forestry University, Harbin Normal University, Heilongjiang University, Northeast Petroleum University and Harbin University.

About 1260 papers from 11 countries and regions were submitted to the conference, and 610 of them were selected for presentation and publication. These selected papers span a wide range of information, computing and telecommunication technologies from theory to applications. Signal processing and electronics engineering are very hot and important research topics, and more and more attentions have been attracted on it in recent years. So, we have selected 7 from the 610 research papers on signal processing and electronics engineering to form this special issue. The quality of the selected papers was very high. The authors of the 7 selected papers promised that the papers were original work.

"Research on Robust Control of Gas Tungsten Arc Welding System with the LMI Approach", by Junfeng Wu, proposes robust control and robust optimal control for sampled-data systems with structured uncertainty.

"Calculation for the Heating and Safe Operation Time of High-voltage Induction Motors of YKK Series in Starting Process", by Yunyan Xia, proposes dynamic equations and wind network model of the medium-sized high-voltage motors.

"Design of a Regular Expression Matching System Based on Network on Chip", by Linhai Cui, proposes an efficient method for Regular Expression Matching (REM) by reusing Intellectual Property (IP) cores in a new architecture of Network on Chip (NoC).

\footnotetext{
${ }^{\S}$ Address correspondence to this editor at the Computer School of Heilongjiang University, China; Tel: +8613945092840; Fax: +86-451-86608587; E-mail: jbli@hlju.edu.cn

${ }^{8}$ Guest Editor

${ }^{\#}$ Co-Guest Editors
}

"A Hybrid Ant Colony Algorithm for Quadratic Assignment Problem", by Guo Hong, proposes characteristics of ant colony algorithm and particle swarm optimization algorithm.

"A Sequence List Algorithm for the Job Shop Scheduling Problem", by Shuli Zhang, proposes the sequence list algorithm for the job shop scheduling problem algorithms.

"An Improved Random Forest Algorithm for ClassImbalanced Data Classification and its Application in PAD Risk Factors Analysis", by Dengju Yao, proposes an improved random forest was proposed based on SMOTE.

"Variable Length Reconfigurable Algorithms and Architectures for DCT/IDCT Based on Modified Unfolded CORDIC", by Hai Huang, proposes a coordinate rotation digital computer (CORDIC) based variable length reconfigurable DCT/IDCT algorithm and corresponding architecture.

Many thanks go to the authors of this special issue for their efforts preparing the manuscripts and for their contributions to scientific research on signal processing and electronics engineering. We would like to thank all the referees of the selected papers for their efforts to ensure the quality of this special issue. Last but not least, we would also like to take this opportunity to appreciate all the members of the editorial board of "The Open Electrical \& Electronic Engineering Journal" for their exceptional efforts to publish this special issue. Finally, we hope you enjoy reading this special issue.

\section{GUEST EDITOR}

Professor Jin-bao Li (1969-) received his bachelor degree in radio electronics from Heilongjiang University, Harbin China in 1991. He received his master degree in Computer Engineering from Heilongjiang University, Harbin China in 1997. He received his doctor degree in the same field in 2007 from Harbin Institute of Technology. Since 1991 he has been concerned with sensor network, database, mobile computing, parallel and distributed computing. Now 


\begin{tabular}{l} 
he is the executive vice president \\
of School of Computer Science \\
and Technology in Heilongjiang \\
University. \\
\hline Professor Shou-xu Jiang (1968-) \\
received his bachelor degree in \\
Computer Engineering from Har- \\
bin Institute of Technology, Har- \\
bin China in 1990. He received his \\
master degree and doctor degree in \\
the same field in 1995 and 2007, \\
respectively. Since 1990 he has \\
been concerned with Pervasive \\
computing, wireless sensor net- \\
works, mobile-aware network, the \\
Internet of things, physical integ- \\
ration of information systems \\
(CPS). Now he is the vice presi- \\
dent of School of Software in \\
Harbin Institute of Technology.
\end{tabular}

Professor Chunxiang Zhang (1974-) received his bachelor's degree from Heilongjiang University in 1998. He received his master's degree and doctor degree in 2002 and 2007. He works as a professor at Harbin University of Science and Technology. His research interests are natural language processing, machine translation and machine learning. He has authored and co-authored more than twenty journal and conference papers in these areas.

(C) Li et al.; Licensee Bentham Open .

This is an open access article licensed under the terms of the Creative Commons Attribution Non-Commercial License (http://creativecommons.org/licenses/by-nc/3.0/) which permits unrestricted, non-commercial use, distribution and reproduction in any medium, provided the work is properly cited. 